\title{
Basic Behavioral and Social Science Research
}

National Cancer Institute

\section{Source}

National Cancer Institute. Basic Behavioral and Social Science Research. NCI Thesaurus.

Code C15769.

Basic social sciences and behavioral research designed to strengthen the understanding of (a) the determinants and processes that influence HIV-related risk behaviors and (b) the consequences of HIV disease. 